Please quote as: Leimeister, J. M. \& Glauner, C. (2008): Hybride Produkte Einordnung und Herausforderungen für die Wirtschaftsinformatik. In: Wirtschaftsinformatik, Ausgabe/Number: 3, Vol. 50, Erscheinungsjahr/Year: 2008. Seiten/Pages: 248-251. 


\section{Hybride Produkte - \\ Einordnung und Herausforderungen für die Wirtschaftsinformatik}

\section{DOI 10.1365/s11576-008-0051-z}

\section{Die Autoren}

\section{Dr. Jan Marco Leimeister \\ Technische Universität München Wirtschaftsinformatik (I 17) \\ Boltzmannstr. 3 \\ 85748 Garching b. München \\ Deutschland \\ leimeister@in.tum.de \\ http://www.infobase.de}

\author{
Christoph Glauner \\ DIN Deutsches Institut für Normung \\ Referat Entwicklungsbegleitende \\ Normung \\ Burggrafenstr. 6 \\ 10787 Berlin \\ Deutschland \\ christoph.glauner@din.de \\ http://www.ebn.din.de
}

Der Beitrag wurde u. a. im Rahmen des vom BMBF geförderten und vom dlr-pt betreuten Verbundvorhabens SPRINT - Systematisches Design zur Integration von Produkt und Dienstleistung - hybride Wertschöpfung (Förderkennzeichen 01FD0609, Projektkoordination: Dr. Jan Marco Leimeister) erstellt.

Eingereicht am 2007-08-17, nach zwei Überarbeitungen angenommen am 2008-02-01 durch Prof. Dr. Sinz.

Unternehmen im produzierenden Bereich sehen sich einem erhöhten Preis- und Wettbewerbsdruck gegenüber. Gewinnmargen liegen bei Produktherstellern immer weniger im physischen Produkt als in dazugehörigen Dienstleistungen. Doch auch Dienstleistungsunternehmen stehen in zunehmendem Wettbewerb. So steigt in vielen Bereichen der Konkurrenzdruck durch neue (insb. internationale) Anbieter am Markt, die bspw. durch Ausnutzung von Lohnkostendifferenzen (Offshoring) starke Kostenvorteile bei ähnlicher Qualität haben.

Um auf diese Wettbewerbssituation zu reagieren, wandeln sich sowohl produzierende Unternehmen als auch Dienstleistungsanbieter selbst zu sog. Lösungsanbietern (vgl. bspw. Reiss und Präuer 2001). Hierdurch verwischen die Grenzen zwischen Produkt(ion) und Dienstleistung immer stärker und es entstehen hybride Wertschöpfungsstrukturen, die sich durch eine Kombination von Sach- und Dienstleistung auszeichnen. Charakteristisch für hybride Produkte ist dabei die intelligente Verzahnung aus physischem Produkt und Dienstleistung, die bereits bei der Konzeption und Entwicklung eng miteinander verbunden werden und deren Einzelkomponenten nur noch schwer voneinander entkoppelt werden können.

Hybride Wertschöpfung verheißt in zweierlei Hinsicht hohes ökonomisches Potenzial. Einerseits kann sie zur Sicherung des Standorts und der Wettbewerbsposition sowie zum Arbeitsplatzerhalt bestehender Unternehmen im Inland beitragen, andererseits eröffnet hybride Wertschöpfung die Chance auf Wachstum durch innovative Produktideen und insbesondere die Erschließung neuer Zielgruppen und Schaffung neuer Märkte.

Die Diskussion zu hybriden Produkten und hybrider Wertschöpfung entwickelte sich sowohl in der Wissenschaft als auch in der Wirtschaft erst in den letzten Jahren. Ausgangspunkt der Diskussion war die Erkenntnis, dass das Angebot produktbegleitender Dienstleistungen, welche vor allem in der Branche des Maschinenbaus in den letzten Jahrzehnten deutlich an Bedeutung gewonnen haben, lediglich ein erster Schritt in diese Richtung ist. Produktbegleitende Dienstleistungen gehören zwar seit jeher zu den Leistungen des verarbeitenden Gewerbes (z. B. bei der Planung und Beratung oder der Schulung des Kunden), erst in den letzten Jahren ist jedoch die strategische Bedeutung dieser Leistungen in den besonderen Fokus von
Wissenschaft und Wirtschaft gelangt (Luczak 2004).

Der Hintergrund der Entwicklung hin zu hybrider Wertschöpfung ist die Erkenntnis, dass Kunden kein Interesse an Produkten oder Dienstleistungen per se haben, sondern sie erwarten vielmehr die Lösung eines Problems, mit dem sie konfrontiert sind, oder die Erfüllung eines Bedarfes, den sie haben. In der Praxis hat sich als Reaktion dieser Erwartungshaltung des Kunden u. a. der Begriff „performance contracting“ etabliert. Dieser Ansatz der Problemlösung ist bislang allerdings nur in wenigen Unternehmen verwirklicht worden (Sawhney 2006).

\section{Hybride Produkte \\ - Begriffserläuterung im Kontext bisheriger Forschungen}

Das Wort „Produkt“ bezeichnet laut Duden ein Erzeugnis oder einen Ertrag sowie nach Langenscheidt auch das Ergebnis einer Bemühung. In der ISO 9000 ist ein Produkt definiert als Ergebnis eines Prozesses. Das Wort „hybrid“ wird im Duden als aus Verschiedenartigem zusammengesetzt, von zweierlei Herkunft; gemischt; zwitterhaft beschrieben. Etwas verallgemeinert bedeutet hybrid also, dass etwas aus mehreren Teilen besteht und diese eng vermischt, sogar zwitterhaft sein können. Die Teile des Ganzen treten also nicht mehr einzeln in Erscheinung, sondern ergeben ein neues Ganzes. Die Eigenschaften der Teile können aber durchaus noch erkennbar sein, was durch die Zwitterhaftigkeit angedeutet wird. Aus diesen beiden Wortbestandteilen lässt sich folgende semantische Annäherung ableiten:

Ein hybrides Produkt ist eine Leistung, die aus mehreren Teilen besteht, welche nicht mehr ohne Weiteres einzeln erkennbar sind, deren unterschiedliche Eigenschaften aber das hybride Produkt prägen. Hybride Wertschöpfung umfasst entspre- 


\begin{tabular}{|c|c|c|}
\hline Begriff & Definition & Verwendung u. a. durch \\
\hline $\begin{array}{l}\text { Produktbegleitende } \\
\text { Dienstleistungen }\end{array}$ & $\begin{array}{l}\text { „Produktbegleitende Dienstleistungen sind immaterielle Leistungen zur Erhöhung } \\
\text { des Kundennutzens und zur Erzeugung von Kundenlösungen, die von Maschinen- und } \\
\text { Anlagenlieferanten unmittelbar oder durch Dritte erbracht werden." }\end{array}$ & VDMA Einheitsblatt 24471 (2003) \\
\hline Performance Contracting & $\begin{array}{l}\text { „Beim Performance Contracting erwirbt der Kunde von einem Hersteller oder } \\
\text { Dienstleister nicht mehr Produkte und einzelne produktbegleitende } \\
\text { Dienstleistungen, wie z. B. Reparaturdienstleistungen, sondern nur noch eine } \\
\text { umfassende Dienstleistung. Diese besteht darin, dass der Anbieter dem Kunden } \\
\text { nicht mehr das Produkt verkauft, sondern die Leistung zur Nutzung zur Verfügung } \\
\text { stellt. Das Geschäftsmodell Performance Contracting kann dabei je nach Umfang der } \\
\text { Leistungsübernahme durch den Anbieter unterschiedliche Formen annehmen“ }\end{array}$ & Backhaus/Kleinkamp (2001) \\
\hline \multirow[t]{3}{*}{ Lösung bzw. Solution } & $\begin{array}{l}\text { „Die Kernleistung von Solutions ist eine Dienstleistung [...]. Dieser Servicekern } \\
\text { wird möglicherweise umkränzt durch Hardwarebestandteile, die jedoch lediglich } \\
\text { flankierend zur Gesamtlösung beitragen. Die Leistungsbestandteile werden } \\
\text { je nach Bedarf hersteller- und branchenübergreifend zusammengestellt." }\end{array}$ & Reiss, Präuer (2001, S. 49) \\
\hline & $\begin{array}{l}\text { "A solution is a combination of products and services that creates value beyond } \\
\text { the sum of its parts [...], it is the level of customization and integration that sets } \\
\text { solutions above products or services or bundle of products and services." }\end{array}$ & $\begin{array}{l}\text { Johansson, Krishnamurthy, } \\
\text { Schlissberg }(2003, \text { S. 118) }\end{array}$ \\
\hline & $\begin{array}{l}\text { "A solution is a customized, integrated combination of products, } \\
\text { services and information that solves a customer's problem." }\end{array}$ & $\begin{array}{l}\text { Sawhney, Wolcott, } \\
\text { Arroniz }(2006, \text { S. 78) }\end{array}$ \\
\hline $\begin{array}{l}\text { Hybride Leistungsbündel } \\
\text { / hybrides Produkt }\end{array}$ & $\begin{array}{l}\text { "Ein hybrides Produkt ist ein Leistungsbündel, das sich aus einer speziell aufeinander } \\
\text { abgestimmten Kombination aus Sach- und Dienstleistungsanteilen zusammensetzt und } \\
\text { eine auf die individuellen Bedürfnisse des Kunden ausgerichtete Problemlösung darstellt." }\end{array}$ & Kersten et al. (2006) \\
\hline Hybride Wertschöpfung & $\begin{array}{l}\text { Wertschöpfung durch hybride Produkte - Kern solcher Strategien sind hybride Produkte (im } \\
\text { Folgenden auch als Lösungen bezeichnet). Darunter sind zu kundenspezifischen Problem- } \\
\text { lösungen integrierte Leistungsbündel aus Sachgütern und Dienstleistungen zu verste- } \\
\text { hen, deren Wert für den Kunden durch die Integration den Wert der Teilleistungen übersteigt. } \\
\text { Software als Teil der Lösung kommt dabei oftmals eine zentrale Integrationsfunktion zu. }\end{array}$ & $\begin{array}{l}\text { Leimeister (2008), aufbauend } \\
\text { auf Böhmann / Krcmar (2006) } \\
\text { und Galbraith (2002). }\end{array}$ \\
\hline
\end{tabular}

chend die Wertschaffung und Wertaneignung mit hybriden Produkten.

In der Literatur findet sich eine Vielzahl an Begriffen, die im Kontext hybrider Produkte Verwendung finden. Eine Auswahl ist in Tab. 1 mitsamt ihrer Definition dargestellt. Neben den hier dargestellten finden sich in Wissenschaft und Wirtschaft noch weitere Begriffe (z. B. Produkt-Service-Systeme), die jedoch keine größere Verbreitung gefunden haben.

Erkennbar wird aus Tab. 1, dass eine klare Abgrenzung zwischen den einzelnen Begriffen in der Literatur nicht stattfindet. Häufig werden in den Abhandlungen verschiedene der oben genannten Begriffe synonym verwandt, beispielsweise „hybride Wertschöpfung“, „Lösungen“ und „Leistungen“ (Böhmann und Krcmar 2006).

\section{Ansätze zur Systematisierung und Typologisierung hybrider Produkte}

Grundlage der wissenschaftlichen Durchdringung und systematischen Gestaltung hybrider Produkte ist die Strukturierung des Problembereiches. Hierzu gilt es, Strukturparameter zu identifizieren und zu analysieren. Aufbauend hierauf kann eine zielgerichtete Gestaltung und Wei- terentwicklung von hybriden Produkten erfolgen. Ein erster Schritt in diese Richtung ist die Systematisierung und Typologisierung hybrider Produkte.

Die bislang in der Literatur am häufigsten verwendete gütertypologische Abgrenzung erfolgt anhand von zwei Dimensionen: die Interaktion mit dem Kunden während des Erstellungsprozesse sowie der Grad der Immaterialität des Leistungsergebnisses. Hybride Produkte können hierbei auf der Achse der Immaterialität des Leistungsergebnisses zwischen materiell und immateriell eingruppiert werden. Die Interaktion mit dem Kunden ist tendenziell eher hoch, kann jedoch vor allem bei hybriden Produkten im Consumer-Bereich auch niedrig sein (vgl. Bild 1).

Diese Unterscheidung zwischen hybriden Produkten, Sachleistungen und Dienstleistungen hilft zwar für das bessere Verständnis - für eine Typologisierung hybrider Produkte selbst ist sie jedoch noch zu undifferenziert. Hierfür stellen Burianek et al. (2007) mit als Erste einen feingranulareren Kategorisierungsansatz hybrider Produkte vor. Es werden auf Basis bestehender Gliederungsansätze aus dem Sach- und Dienstleistungsbereich insgesamt sieben Kriterien vorgeschlagen, nach denen hybride Produkte auf Grundlage ihrer Komplexität charakterisiert werden können:
- Art des Kundennutzens (produktorientiert $\leftrightarrow \rightarrow$ ergebnisorientiert)

- Umfang des Leistungsangebotes (partiell $\leftrightarrow \rightarrow$ umfassend)

- Anzahl/Heterogenität der Teilleistungen (gering $\leftrightarrow \rightarrow$ hoch)

- Grad der technischen Integration (niedrig $\leftrightarrow$ hoch)

- Grad der Integration in die Wertschöpfungsdomäne des Kunden (niedrig $\leftrightarrow$ hoch)

- Grad der Individualisierung (niedrig $\leftrightarrow$ hoch)

- Zeitliche Dynamik/Veränderlichkeit der Leistungserbringung (gering $\leftrightarrow \rightarrow$ hoch).

Ähnlich der Typologisierung von Leistungsbündeln nach Engelhardt et al. (1993) liegt auch diesem Ansatz der Gedanke zugrunde, dass die Komplexität nicht nur über zwei Extremausprägungen („gering“ bzw. „hoch“) verfügt, sondern es sich vielmehr um ein Kontinuum handelt, das sich von "gering“ bis „hoch“ erstreckt (dimensionale Eigenschaft). Ferner ist anzumerken, dass die Merkmale nicht vollständig unabhängig voneinander sind, sondern sich im Einzelfall gegenseitig beeinflussen können.

Die Ausprägungen der einzelnen Merkmale der Kriterien haben einen erheblichen Einfluss auf die Komplexität des jeweiligen hybriden Produktes und damit 


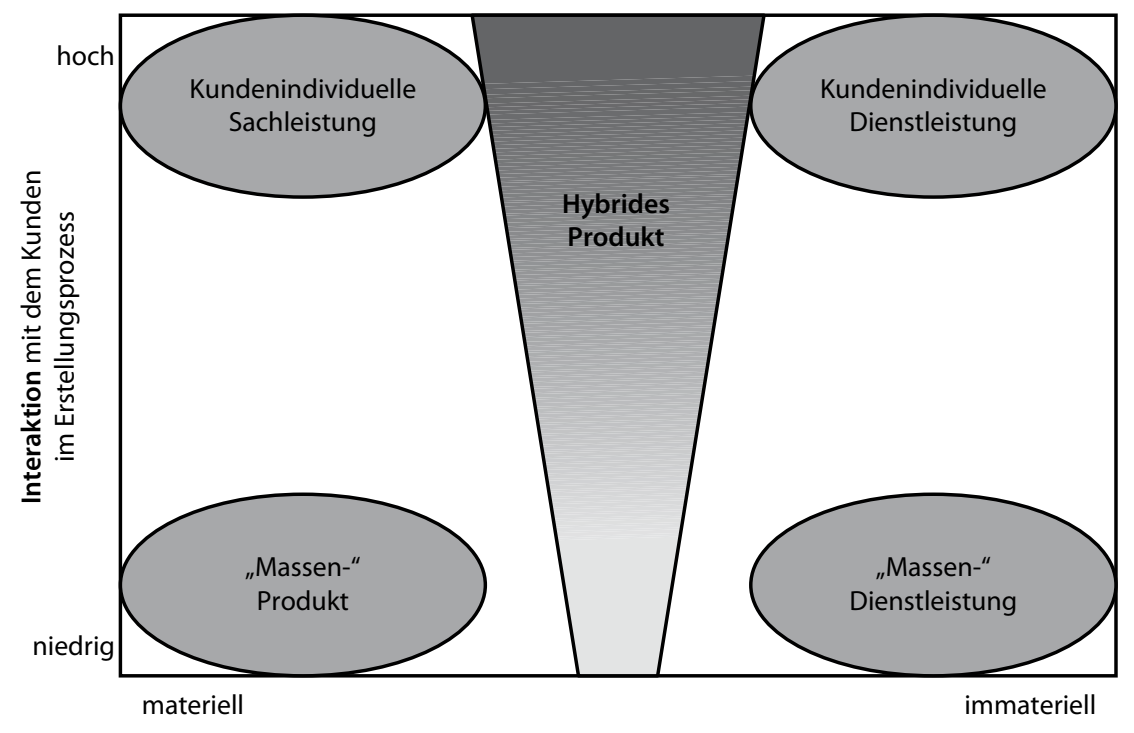

Grad der Immaterialität des Leistungsergebnisses

Bild 1 Gütertypologische Abgrenzung hybrider Produkte (in Anlehnung an Engelhardt et al. 1993, S. 417)

auf die Leistungserstellung. Beispielsweise ist die Komplexität der Leistungserstellung bei einer kleinen Zahl von relativ homogen Teilleistungen (z. B. IT-gestütztes Facility Management in einem reinen Bürogebäude), viel geringer als bei einer großen Zahl an heterogenen Teilleistungen (z. B. IT-gestütztes Facility Management in einem Industriekomplex mit unterschiedlichsten Bürogebäuden, technischen Anlagen etc.). Eine Unterscheidung in unterschiedliche Komplexitätsstufen erscheint daher sinnvoll, da hierdurch und insb. mit auf die einzelnen Stufen abgestimmten Gestaltungsempfehlungen eine Hilfestellung zur Komplexitätsreduktion und -bewältigung gegeben wird.

\section{Bedeutung für die Wirtschaftsinformatik und Konsequenzen für die Forschung}

Trotz des für zahlreiche Unternehmen fundamentalen Perspektivenwechsels und der großen Bedeutung der Integration von Sachgütern und Dienstleistungen ist der Forschungsstand hierzu - sicher auch im Zuge der Neuheit dieses Themenfeldes als unzureichend anzusehen. Die größte Herausforderung wird dabei weniger in innovativen Ideen als bei der Organisation des Managements dieser Lösungen gesehen (Böhmann und Krcmar 2007).

In der Praxis lässt sich beispielsweise beobachten, dass Sach- und Dienstleistun- gen meist getrennt entwickelt und Dienstleistungen nur „produktbegleitend“ zum bisherigen Wertschöpfungsprozess der Unternehmen hinzugefügt werden. Auch in der Forschung wurde die Wertschöpfung von Sachgütern und Dienstleistungen bislang oftmals getrennt oder bestenfalls als Bündelung bereits bestehender Komponenten betrachtet. So gibt es detaillierte Ansätze zur Entwicklung von physischen Produkten (bspw. Lindemann 2006) und seit einiger Zeit auch zur systematischen Entwicklung von Dienstleistungen (vgl. bspw. Herrmann et al. 2004; Scheer und Spath 2004; Bullinger und Scheer 2003). Eine integrierte Betrachtung hybrider Produkte ist jedoch bislang kaum vollzogen worden.

Ein Mangel besteht bereits bei grundlegenden Fragestellungen, wie z. B. die typenbezogene Einordnung und Abgrenzung hybrider Produkte. Ferner sind Interdependenzen zwischen den einzelnen konstituierenden Kriterien nicht berücksichtigt oder gar empirisch überprüft.

Darauf aufbauend besteht ein großer Bedarf nach praktischen Handlungsempfehlungen. Gestaltungsempfehlungen setzen dezidiertes Wissen über Mittel-ZielBeziehungen voraus. Für die Entwicklung und Erbringung hybrider Produkte erfordert dies fundierte Kenntnisse über den Zusammenhang zwischen Ausprägungen hybrider Produkte - verstanden als Summe aller Strukturparameter für die Gestaltung -, den entsprechenden Wertschaffungs- und Wertaneignungsprozes- sen und der daraus resultierenden Ergebnisqualität. Hierzu bedarf es entsprechender Bezugsrahmen und Referenzmodelle, die auch einer empirischen Überprüfung Stand halten. Vielversprechend ist hierbei insbesondere die informationstechnische Unterstützung der unternehmensinternen wie auch -übergreifenden Prozesse. Dies umfasst zuallererst die Definition von Grundlagen, die u. a. die Kommunikation zwischen Produzenten, Dienstleistern und Kunden erleichtern. Zentral ist die Entwicklung von Prozessmodellen und Vorgehensweisen, um unternehmensinterne Prozesse sowie unternehmensübergreifende Transaktionen zu vereinfachen und transparenter zu gestalten. Weitere relevante Fragestellungen finden sich bei unterschiedlichen Strategien der Wertaneignung (bspw. Pricing, etc.) im Bereich der hybriden Wertschöpfung.

Ferner scheint auch die Rolle von Informationssystemen und Informationstechnologie zur Produkt-Dienstleistungsbündelung (bspw. als Teil der hybriden Lösung oder als Infrastruktur zur Leistungserbringung) noch nicht hinreichend verstanden. Neben geeigneten GovernanceProzessen und -Strukturen sind für deren entsprechende Implementierung in Unternehmen die Rolle von leistungsfähigen ITInfrastrukturen zur Unterstützung der hybriden Wertschöpfung zu untersuchen, da hierdurch bspw. die notwendigen Informationen gebündelt und gezielt zur Weiterverarbeitung aufbereitet werden können. Insgesamt können also im Themenfeld der hybriden Wertschöpfung von informationstechnischer Seite wichtige Impulse gegeben werden.

Notwendig erscheint zudem die frühzeitige Entwicklung von Normen und Standards, um beispielsweise Transparenz zu gewährleisten oder grundlegende Prozesse und Schnittstellen (besonders im ITBereich) zu vereinheitlichen. Normen und Standards können zudem dabei unterstützen, die in der Wirtschaftsinformatik entwickelten Lösungen bezüglich der Entwicklung und Erbringung hybrider Produkte schneller in die Anwendung zu tragen (Behrens und Blind 2007).

Hybride Produkte und darauf aufbauend hybride Wertschöpfung erfordern einen Paradigmenwechsel hin zu einer ganzheitlichen, am Kundenbedarf ausgerichteten Gesamtlösungsorientierung. Dies ist eine große Herausforderung für Wissenschaft und Praxis - es muss eine 
Integration bereits existierender Erkenntnisse und Ansätze aus unterschiedlichen Wissenschaftsdisziplinen geleistet werden. Diese Integrationsperspektive anzubieten und diese Weiterentwicklungsleistung zu erbringen, ist eine Chance für die Wirtschaftsinformatik.

\section{Literatur}

Backhaus, K.; Kleinkamp, C. (2001): Marketing von investiven Dienstleistungen. In: Bruhn, M.; Meffert, $H$. (Hrsg.), Handbuch Dienstleistungsmanagement. 2. Aufl., Gabler, Wiesbaden, S. 73-101.

Behrens, Hermann; Blind, Knut (2007): Stärkung der Integration von Innovation und Normung. Fraunhofer IRB Verlag, Stuttgart.

Böhmann, Tilo; Krcmar, Helmut (2006): Komplexitätsmanagement als Herausforderung hybrider Wertschöpfung im Netzwerk. In: Wojda, Franz; Barth, Alfred (Hrsg.): Innovative Kooperationsnetzwerke. DUV, Wiesbaden, S. 81-106.

Böhmann, Tilo; Krcmar, Helmut (2007): Hybride Produkte: Merkmale und Herausforderungen. In: Bruhn, Manfred; Stauss, Bernd (Hrsg.): Wertschöpfungsprozesse bei Dienstleistungen - Forum Dienstleistungsmanagement. Gabler, Wiesbaden, S. 239-258.

Bullinger, Hans-Jörg; Scheer, August-Wilhelm (Hrsg.) (2003): Service Engineering - Entwicklung und Gestaltung innovativer Dienstleistungen. Springer Verlag, Berlin, Heidelberg, New York.

Burianek, Ferdinand; Ihl, Christoph; Bonnemeier, Sebastian; Reichwald, Ralf (2007): Typologisierung hybrider Produkte. Ein Ansatz basierend auf der Komplexität der Leistungserbringung. Arbeitsbericht Nr. 01/2007 des Lehrstuhls für Betriebswirtschaftslehre - Information, Organisation und Management der TU München.

Engelhardt, Werner H.; Kleinaltenkamp, Michael; Reckenfelderbäumer, Martin (1993): Leistungsbündel als Absatzobjekte. In: Zeitschrift für Betriebswirtschaftliche Forschung 45 (5), S. 394-426.

Galbraith, Jay (2002). Organizing to deliver solutions. In: Organizational Dynamics, 31(2), S. 194-207.

Herrmann, Thomas; Kleinbeck, Uwe; Krcmar, Helmut (Hrsg.) (2004): Konzepte für das Service-Engineering: Modularisierung, Prozessgestaltung und Produktivitätsmanagement. Physica Verlag, Heidelberg.

Johansson, Juliet E.; Krishnamurthy, Chandru; Schlissberg, Hank E. (2003): Solving the solutions problem. In: McKinsey Quarterly (3), S. 116-125.

Kersten, Wolfgang; Zink, Thomas; Kern, Eva-Maria (2006): Wertschöpfungsnetzwerke zur Entwicklung und Produktion hybrider Produkte: Ansatzpunkte und Forschungsbedarf. In: Blecker, Thorsten; Gemünden, Hans G. (Hrsg.): Wertschöpfungsnetzwerke. Festschrift für
Bernd Kaluza. Erich Schmidt Verlag, Berlin, S. 189-202.

Leimeister, Jan Marco (2008): Hybride Wertschöpfung in der Gesundheitswirtschaft. Kumulative Habilitationsschrift, Technische Universität München.

Lindemann, Udo (2006): Methodische Entwicklung technischer Produkte. Methoden flexibel und situationsgerecht anwenden: Methoden Flexibel und Situationsgerecht Anwenden. 2. Aufl., Springer, Berlin.

Luczak, Holger (Hrsg.) (2004): Betriebliche Tertiarisierung - Der ganzheitliche Wandel vom Produktionsbetrieb zum dienstleistenden Problemlöser. DUV, Wiesbaden.

Reiss, Michael; Präuer, Arndt (2001): Solutions Providing: Was ist Vision - was Wirklichkeit? In: Absatzwirtschaft 44 (5), S. 48-53.

Sawhney, Mohanbir (2006): Going beyond the Product: Defining, Designing, and Delivering Customer Solutions. In: Lusch, Robert F.; Vargo, Stephen L. (Hrsg.): The Service-dominant Logic of Marketing. M. E. Sharpe, Armonk, S. 365-380.

Sawhney, Mohanbir; Wolcott, Robert; Arroniz, Inigo (2006): The 12 Different Ways for Companies to Innovate. MIT Sloan Management Review, Spring 2006.

Scheer, August-Wilhelm; Spath, Dieter (Hrsg.) (2004): Computer Aided Service Engineering. Springer Verlag, Berlin.
WWW. VIEWEGTEUBNER, DE

Investitions-

management mit SAP $^{\circledR}$ praktisch umsetzen

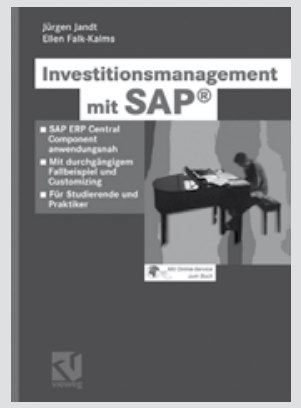

Jürgen Jandt | Ellen Falk-Kalms

\section{Investitions-}

management mit SAP ${ }^{\circledR}$

SAP ERP Central Component anwendungsnah. Mit durchgängigem Fallbeispiel und Customizing. Für Studierende und Praktiker 2008. XV, 474 S. mit 159 Abb. mit Online Service Br. EUR 44,90 ISBN 978-3-8348-0267-5

Unter Einsatz des Applikationssystems SAP werden die einzelnen Teilschritte des Investitionsmanagements vorgestellt. Eine Fallstudie begleitet das Investitionsmanagement. Das Buch ermuntert zum Selbststudium und leitet zum praktischen betrieblichen Einsatz an.

\section{Fax $+49(0) 611.7878-420$}

\section{Ja, ich bestelle} Exemplare Investitionsmanagement mit SAP ISBN 978-3-8348-0267-5 EUR 44,90

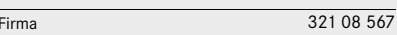

Name, Vorname

Abteilung

Straße (bitte kein Postfach)

PLZ | Ort

Datum | Unterschrift

Geschäftsführer: Dr. Ralf Birkelbach, Albrecht F. Schirmacher AG Wiesbaden HRB 9754 